\title{
Cuidados de Enfermagem prestados à criança portadora de mielomeningocele e suas complicações
}

Nursing Care provided to children with myelomeningocele and its complications

\author{
Atención de enfermería a niños con mielomeningocele y sus complicaciones \\ Rhabech da Silva Vieira ${ }^{1 *}$, Catia Maria Diogo ${ }^{2}$, Carolina de Lourdes Julião Vieira ${ }^{3}$, Jannaína Sther \\ Leite Godinho Silva ${ }^{4}$, José Carlos do Nascimento ${ }^{5}$, Marilei de Melo Tavares ${ }^{6}$
}

Como citar esse artigo. Vieira, RS;

Diogo; CM; Vieira, CLJ; Silva, JSLG; do

Nascimento, JC; Tavares, MM. Cuidados de

Enfermagem prestados à criança portadora

de mielomeningocele e suas complicações.

Revista Pró-UniverSUS. 2021 Jul./Dez; 12 (2)

SUPLEMENTO: 94 - 101

\section{Resumo}

A Mielomeningocele (MMC) é uma doença congênita caracterizada por um Defeito no Fechamento do Tubo Neural (DFTN). No geral, essa patologia afeta de maneira significativa a vida de seus portadores e de seus familiares, atuando de forma negativa no desenvolvimento psicomotor e também é fator responsável para que o portador adquira outras patologias descritas ao longo do estudo apresentado. As patologias congênitas adquiridas a partir da mielomeningocele são incapacitantes e fatais se não tratadas de forma correta e precoce. Dessa forma, o presente estudo tem o objetivo de analisar a assistência prestada pela equipe de enfermagem à criança portadora de mielomeningocele e suas complicações. Para tal, foi realizada uma reflexão teórica com abordagem qualitativa com base na revisão literária de artigos científicos em bases de dados. Sabe-se que o cuidado pela equipe de enfermagem é integral e tem início na Atenção Primária de Saúde, no entanto, por se tratar de uma gestação de alto risco, a gestante é encaminhada ao atendimento na alta complexidade e novamente a equipe de enfermagem realiza a assistência. Desde a descoberta da gestação à alta hospitalar é a equipe de enfermagem que mantém o maior contato possível com os pacientes e, por isso, se dá a importância de analisar e definir a melhor maneira de se prestar tal cuidado.

Palavras-chave: Cuidados de Enfermagem; Espinha Bífida; Mielomeningocele; Recém-Nascido..

\begin{abstract}
Myelomeningocele (MMC) is a congenital disease characterized by a Neural Tube Closure Defect (DFTN). In general, this pathology significantly affects the lives of its patients and their families, acting negatively on psychomotor development and is also a responsible factor for the patient to acquire other pathologies described throughout the study presented. Congenital pathologies acquired from myelomeningocele are disabling and fatal if not treated correctly and early. Thus, the present study aims to analyze the assistance provided by the nursing team to children with myelomeningocele and its complications. To this end, a theoretical reflection with a qualitative approach was carried out based on the literary review of scientific articles in databases. It is known that care by the nursing team is integral and begins in Primary Health Care, however, because it is a high-risk pregnancy, the pregnant woman is referred to care in high complexity and again the nursing team performs assistance. From the discovery of pregnancy to hospital discharge, it is the nursing team that maintains the greatest possible contact with patients and, therefore, the importance of analyzing and defining the best way of providing such care is given.
\end{abstract}

Keywords: Nursing Care; Spina Bifida; Myelomeningocele; Newborn.

${ }^{1 *}$ Discente do curso de Enfermagem / Universidade de Vassouras, RJ, Brasil. E-mail: rhabechvieira14@gmail.com ORCID: https://orcid.org/0000-0002-1780-2937

${ }^{2}$ Doutora. Docente do curso de Enfermagem / Universidade de Vassouras, RJ, Brasil. E-mail: cmdiogo966@gmail.com ORCID: https://orcid.org/0000-0002-7000-5961

${ }^{3}$ Doutora. Docente do curso de Enfermagem / Universidade de Vassouras, RJ, Brasil. E-mail: caroljuliaovieira@gmail.com ORCID: https://orcid.org/0000-0002-5785-5988 ${ }^{4}$ Mestre. Docente do curso de Enfermagem / Universidade de Vassouras, RJ, Brasil. E-mail: jjasther@gmail.com ORCID: https://orcid.org/0000-0002-8308-2093

${ }^{5}$ Mestre. Docente do curso de Enfermagem / Universidade de Vassouras, RJ, Brasil. E-mail: josecarlosdonascimento28@gmail.com ORCID: https://orcid.org/0000-0002-4345-2707 ${ }^{6}$ Pós-Doutora pela UERJ. Docente do Curso de Enfermagem da Universidade de Vassouras. Vassouras, RJ, Brasil. E-mail: marileimts@hotmail.com ORCID: http://orcid.org/00000002-3276-0026. 


\section{Resumen}

El mielomeningocele (MMC) es una enfermedad congénita caracterizada por un defecto de cierre del tubo neural (DFTN). En general, esta patología afecta significativamente la vida de sus pacientes y sus familias, actuando negativamente sobre el desarrollo psicomotor y es también un factor responsable de que el paciente adquiera otras patologías descritas a lo largo del estudio presentado. Las patologías congénitas adquiridas por mielomeningocele son incapacitantes y fatales si no se tratan correctamente y en forma temprana. Así, el presente estudio tiene como objetivo analizar la asistencia brindada por el equipo de enfermería a los niños con mielomeningocele y sus complicaciones. Para ello, se realizó una reflexión teórica con enfoque cualitativo a partir de la revisión literaria de artículos científicos en bases de datos. Se sabe que la atención por parte del equipo de enfermería es integral y comienza en Atención Primaria de Salud, sin embargo, por ser un embarazo de alto riesgo, la gestante es referida a cuidados de alta complejidad y nuevamente el equipo de enfermería realiza asistencia. Desde el descubrimiento del embarazo hasta el alta hospitalaria, es el equipo de enfermería el que mantiene el mayor contacto posible con las pacientes y, por ello, se da la importancia de analizar y definir la mejor forma de brindar dicha atención.

Palabras clave: Cuidado de Enfermera; Espina Bífida; Mielomeningocele; Recién Nacido.

\section{Introdução}

A mielomeningocele (MMC) trata-se de um Defeito no Fechamento do Tubo Neural (DFTN) ocasionada por uma falha na oclusão adequada durante a embriogênese por volta da quarta semana de gestação. ${ }^{1}$ Por se tratar de uma patologia pouco conhecida, a porcentagem de nascidos vivos com MMC ainda é elevada estima-se que uma a cada oitocentas crianças nasça com essa malformação. ${ }^{2}$ Diversos estudos já foram realizados, porém nenhum foi capaz de definir com propriedade a verdadeira causa da doença.

Dessa forma, pode-se estabelecer que se trata de uma patologia de etiologia não esclarecida, porém pesquisas apontam a carência de ácido fólico como o fator principal. Sabe-se, através de estudos, que a suplementação periconcepcional e durante o primeiro trimestre de gestação tem reduzido a ocorrência e a recorrência de DFTN. ${ }^{3}$ É recomendado pelo Centro de Controle de Doenças (CDC) o uso de $0,4 \mathrm{mg} /$ dia para mulheres em idade reprodutiva por 3 meses antes da concepção e, para as mulheres que são mães de filhos portadores de DFTN ou que apresentam risco aumentado é recomendado $0,4 \mathrm{mg} / \mathrm{dia}$ e, se planejarem gestação, a dose é aumentada para $4 \mathrm{mg} / \mathrm{dia}^{4}{ }^{4}$

A MMC é apresentada como uma espinha bífida oculta ou cística. A protusão oculta, geralmente, não causa nenhuma sintomatologia, pois não envolve a medula espinhal ou as meninges. A protusão cística é a forma mais grave da doença, uma vez que contém o tecido nervoso exposto não coberto por pele, sua exposição pode comprometer toda a extensão do tubo neural ou limitar-se a uma área.5 O diagnóstico é estabelecido, principalmente, através da ultrassonografia realizada no pré-natal. Além disso, a presença de alfafetoproteína na corrente sanguínea materna também pode ser considerada um fator para diagnóstico de DFTN, porém não é específica para mielomeningocele.

A gravidade das sequelas está relacionada ao tamanho e ao local onde houve a falha no fechamento do tubo neural: lesões localizadas entre as vértebras lombares são características de danos menores ou mais leves. De modo geral, a criança que apresenta mielomeningocele desenvolve distúrbios neurológicos, motores e renais.

Com o avanço da tecnologia o tratamento para a MMC pode ser realizado intra-útero entre a $19^{\mathrm{a}}$ e $27^{\mathrm{a}}$ semana de gestação ${ }^{5}$, porém, caso não seja indicada a cirurgia fetal, a correção poderá ser feita após o nascimento. Nos dois casos é indispensável o trabalho multidisciplinar, envolvendo equipe médica cirúrgica de pediatria e neurologia e equipe de enfermagem.

O recém-nascido que acaba de passar por uma cirurgia neurológica necessita de cuidados específicos e intensos, por isso, a Unidade de Terapia Intensiva Neonatal é o local ideal para que a assistência seja prestada da melhor maneira possível. A equipe de enfermagem exerce um dos papeis mais importantes nesse contexto, pois garante o cuidado integral ao indivíduo ali estabelecido. ${ }^{6}$ Sendo assim, o objetivo do presente estudo é analisar a assistência prestada pela equipe de enfermagem à criança portadora de mielomeningocele e suas complicações.

\section{Materiais e Métodos}

Trata-se de uma reflexão teórica com abordagem qualitativa com base na revisão literária deartigos científicos encontrados nas bases de dados: Literatura Latino Americana e do Caribe em Ciências da Saúde (LILACS) e Scientific Eletronic Library Online (SciELO). Para as pesquisas foram usados os seguintes descritores:recém-nascido, mielomeningocele, cuidados de enfermagem e espinha bifida. Por se tratar de um tema de difícil abordagem e poucos estudos publicados não foi possível estabelecer um recorte temporal.

"Pesquisa qualitativa é o que se aplica ao estudo 
da história, relações e interpretações, crenças, opiniões e percepções do ser humano, permite desvendar os processos sociais para análises de discursos e documentos." 7:21-22.

\section{Resultados e Discussão}

A mielomeningocele é responsável por algumas complicações que podem comprometer a qualidade de vida de seus portadores, algumas delas se não tratadas ou diagnosticadas tardiamente podem levar à morte. A seguir serão descritas as complicações mais presentes, seus métodos de diagnósticos e tratamentos. Além disso, será analisada e discutida a importância que a equipe de enfermagem tem em todas as etapas de vida da criança portadora, desde o pré-natal até a alta hospitalar.

\section{Hidrocefalia}

O cérebro humano produz constantemente um líquido chamado líquido cefalorraquidiano ou líquor que tem entre suas principais funções a capacidade de proteger o Sistema Nervoso Central a partir de uma barreira mecânica e imunológica. A hidrocefalia é o nome dado ao processo em que, por algum motivo, o líquido passa a ser produzido em excesso, comprometendo o bom funcionamento do cérebro e aumentando a pressão intracraniana.

Sabe-se que existem três tipos de hidrocefalia e que estão diretamente ligadas as suas causas: congênita, adquirida e de pressão normal ${ }^{8}$.No caso da mielomeningocele a hidrocefalia é de razão congênita, pois está presente no nascimento, podendo até mesmo ser diagnosticada no período pré-natal. Em uma situação de normalidade, o líquor é produzido no interior do cérebro, circula entre os ventrículos e é reabsorvido em sua parte mais externa, no caso da hidrocefalia congênita há uma dificuldade ou bloqueio total na circulação do líquido causando o aumento do mesmo. Estima-se que 1 a cada 1000 bebês nascem com a hidrocefalia congênita. ${ }^{9}$

Os portadores de hidrocefalia congênita têm uma qualidade de vida comprometida, pois algumas manifestações podem dificultar seu desenvolvimento. Alguns sinais e sintomas podem ser percebidos na criança com hidrocefalia logo ao nascer, como: aumento do perímetro cefálico, pele espessa e brilhante com boa visualização das veias e fontanelas mais rígidas. Além disso, percebe-se posteriormente a dificuldade na alimentação, vômitos frequentes, irritabilidade, sonolência, dificuldades no aprendizado e desenvolvimento da criança.

O tratamento precoce gera uma melhora na expectativa de vida, uma vez que reduz os danos causados pela pressão intracraniana. Esse tratamento é cirúrgico e consiste na instalação de uma derivação ventrículoperitoneal (DVP) no cérebro que drena o líquido para outra parte do corpo, nesse caso, o abdômen ${ }^{10}$. É necessário que haja uma atenção redobrada ao paciente portador da DVP, atentando para sinais de infecções no local da implantação e para obstrução da válvula, geralmente manifestados a partir de vômitos, cefaleia, convulsões e sonolência. No geral, a criança portadora da DVP não precisa de cuidados além dos citados acima, pois isso não é um fator incapacitante para nenhuma atividade de vida normal. O acompanhamento médico deve ser rotineiro, bem como a realização de exames de imagem para prosseguir com a eficácia do tratamento realizado.

\section{Malformação de Arnold Chiari Tipo II}

A malformação de Arnold Chiari tipo II está presente em todas as crianças portadoras de mielomeningocele, porém nem todas apresentam sintomas. Quando sintomáticas as taxas de mortalidade tornam-se elevadas. ${ }^{11}$

Essa doença é representada a partir da herniação do IV ventrículo e outros componentes cerebelares através do forame occipital. Os sinais e sintomas são demonstrados pelo excesso do líquido cefalorraquidiano e, com isso, o aumento da pressão intracraniana, por isso, deve-se certificar que pacientes com hidrocefalia estejam previamente derivados e com o sistema estável, ao excluir a possibilidade de hidrocefalia a cirurgia de descompressão deve ser indicada. O tratamento é estritamente cirúrgico de caráter emergencial para a descompressão posterior ao nível da $\mathrm{C} 1 .{ }^{12}$ Como em praticamente todos os casos, quanto antes o diagnóstico melhor será o prognóstico

\section{Bexiga neurogênica}

O controle da micção envolve atos involuntários e voluntárioscaracterizados, respectivamente, pelo enchimento, armazenamento e esvaziamento da bexiga através da uretra para ambos os sexos. Podendo ser descrita, como:

[...]a micção normal está sujeita a mecanismos voluntários e involuntários, dependentes de centros nervosos que se escalonam desde o córtex cerebral até o plexo-intrínseco da parte vesical. Assim, qualquer lesão nervosa que interfira nesses mecanismos causará modificação no funcionamento da bexiga. Teremos então uma disfunção vesical de origem neurológica, ou uma "bexiga neurogênica. ${ }^{13: 52}$

O termo Bexiga Neurogênica (BN) é usado para representar diversas alterações no controle vesicoesfincterianas, podendo ser de origem congênita ou 
adquirida a partir de um trauma ou de uma doença generativa. Estima-se que a Mielomeningocele seja a mielodisplasia responsável por $90 \%$ dos casos de Bexiga Neurogênica. ${ }^{14} \mathrm{O}$ diagnóstico e a gravidade da lesão é feito após o exame urodinâmico, a partir dele é que serão traçados o prognóstico e o tratamento da patologia.

O tratamento varia de acordo com a gravidade da lesão no trato urinário, podendo ser apenas farmacológico e de derivação de cateterismo intermitente ou cirúrgico a partir da realização de uma derivação externa. ${ }^{14}$

\section{Atraso neuropsicomotor e alterações na anatomia dos membros inferiores}

As crianças portadoras de mielomeningocele necessitam de um cuidado especial desde a gestação e deve ser mantido por um bom período após o nascimento. Atrasos neurológicos são percebidos a partir dos primeiros meses de vida, uma vez que portadores de MMC não se desenvolvem como o esperado. Os atrasos motores também são descobertos da mesma forma, geralmente, possuem alterações na anatomia dos membros inferiores o que impossibilita a progressão do desenvolvimento esperado para a idade.

Um estudo realizado em 2009 identificou que das 42 crianças portadoras de MMC $8(19 \%)$ tinham o segmento torácico afetado, $11(26,2 \%)$ tinham o segmento lombar alto afetado, $16(38,1 \%)$ o segmento lombar baixo afetado e $7(16,7 \%)$ o segmento sacral afetado. ${ }^{15}$ Ainda no mesmo estudo foi identificada a associação da luxação de quadril com o segmento lombar alto afetado e a fratura com o segmento torácico afetado. Uma série de deformações anatômicas também são relatadas em outros estudos analisados, a maioria delas são apresentadas nos membros inferiores, o que dificulta ainda mais a locomoção dos portadores de MMC. De modo geral, lesões torácicas propiciam paralisias de membros inferiores em maior probabilidade. Em contrapartida, a probabilidade de ficar de pé e/ou deambular com auxílio é maior quando o nível anatômico lesionado é mais caudal. ${ }^{16}$

$\mathrm{O}$ atraso no desenvolvimento intelectual se deve principalmente à hidrocefalia, porém muitas crianças apresentam desenvolvimento cognitivo normal ou próximo dele. Os fatores socioeconômicos e ambientais são umas das principais condições para que haja progressão no desempenho neuropsicomotor. ${ }^{16}$

\section{Assistência de enfermagem}

A Enfermagem é uma das profissões que lida diretamente com o ser humano todos os dias. Ao analisar o cotidiano de um hospital, pode-se perceber que a equipe de enfermagem é quem está em contato diariamente com o paciente, desde sua admissão até sua alta hospitalar. A descoberta de uma gestação por si só gera muitas expectativas, receber o diagnóstico que o bebê é portador de uma doença pouco conhecida, de etiologia não esclarecedora e complicações que podem levar à morte é ainda mais difícil para a mãe e para toda família.

No caso da mielomeningocele, a equipe de saúde multidisciplinar exerce um papel fundamental no cuidado prestado aos portadores da patologia e à família. Por se tratar de uma gestação de alto risco, a gestante é encaminhada aos serviços hospitalares ainda no pré-natal, uma vez que durante o parto serão necessários serviços especializados. Sendo assim, desde o encaminhamento ao cuidado de alto risco a equipe de enfermagem estará presente para prestar a assistência necessária.

No dia do parto, é necessário que haja um cuidado maior voltado para a gestante, realizando um momento de escuta dos seus medos, inseguranças, ansiedade buscando sempre acalmá-la para que a hora do nascimento do seu bebê seja prazerosa para os dois. O momento se torna atípico em razão da MMC, mas esse fator não impede que o cuidado integral seja voltado para o bem estar da criança e da mãe.

\section{Sala de parto}

Através da discussão realizada sobre as complicações da mielomeningocele, sabe-se que as correções da protrusão cística e da hidrocefalia são realizadas de maneira cirúrgica o mais rápido possível para que a recuperação seja melhor. A equipe médica é quem avalia se as cirurgias serão no pós-parto imediato ou se aguardarão algum tempo para realizar mais exames e observar possíveis intercorrências. De qualquer maneira, assim que o bebê nasce a equipe de enfermagem e o pediatra ficam responsáveis pelos cuidados ao neonato.

Os bebês portadores de mielomeningocele necessitam de cuidados imediatos diferentes daqueles prestados aos neonatos sem nenhuma anomalia congênita. Devem ser manuseados de forma extremamente cuidadosa, sempre em decúbito lateral e o profissional deve observar as características da membrana que recobre o saco herniário localizado na região dorsal. Após a observação é necessário que se faça um isolamento asséptico na lesão com compressa estéril e plástico poroso a fim de evitar contaminações. ${ }^{17}$

Além dos cuidados prestados acima, como em qualquer outro nascimento, outros cuidados imediatos ao recém-nascido devem ser realizados e são eles: desobstrução das vias aéreas, secar e aquecer, avaliação da vitalidade através do índice de APGAR, verificação 
dos sinais vitais, se possível, realizar clampeamento tardio do cordão umbilical, administração de vitamina $\mathrm{K}$ e vacina de Hepatite B. Os cuidados que se seguem são mais específicos para bebês portadores de MMC e vai depender da conduta médica tomada. ${ }^{17}$

\section{Unidade de Terapia Intensiva Neonatal}

Admite-se a partir daqui o bebê que já realizou a cirurgia de correção da MMC, implantou a DVP para corrigir a hidrocefalia e foi avaliado pelo urologista e recebeu o diagnóstico da bexiga neurogênica. Portanto, os cuidados analisados adiante serão voltados para a boa recuperação cirúrgica e observação de sinais de infecções ou de intercorrências pós-cirúrgicas e orientações aos responsáveis.

Entende-se por Unidade de Terapia Intensiva Neonatal, o "serviço de internação responsável pelo cuidado integral ao recém-nascido grave ou potencialmente grave, dotado de estruturas assistenciais que possuam condições técnicas adequadas à prestação de assistência especializada, incluindo instalações físicas, equipamentos e recursos humanos." ${ }^{\text {18 }}$

Durante a internação na UTI o recém-nascido é assistido por toda a equipe de saúde que está estabelecida no local de forma que seja acompanhado $24 \mathrm{~h}$ enquanto durar sua estadia no hospital. A equipe de enfermagem é responsável pelo cuidado integral, realizando procedimentos e observações que serão imprescindíveis na recuperação do RN. É importante, além de realizar, explicar para a mãe ou o responsável que acompanha o paciente sobre as técnicas que serão realizadas na prestação do cuidado. A criança portadora de MMC requer cuidados por um longo período que será realizado no domicílio por uma pessoa instruída.

Considerando o pós-cirúrgico da MMC realizado, preferencialmente, em até 72 horas após o nascimento, deve-se orientar aos responsáveis sobre a realização do curativo na ferida operatória. Orienta-se quanto à realização asséptica para que não haja contaminação e infecção no local da lesão. As mesmas orientações serão dadas quando a ferida operatória for na região da cabeça e do abdômen para colocação da Derivação VentrículoPeritoneal. É muito importante a prevenção da infecção, uma vez que, além de melhorar o prognóstico, também diminui o tempo da estadia na UTI.

A avaliação do urologista quanto à bexiga neurogênica é que vai direcionar quanto às medidas que serão tomadas para o tratamento. Caso a conduta seja apenas farmacológica sem indicação de uso de sondagem para alívio as orientações aos responsáveis serão as básicas de um tratamento com uso de remédios. Ao adotar o tratamento que se faz necessário o uso de sonda intermitente de alívio é muito importante que o profissional que for realizar o procedimento explique detalhadamente ao responsável que acompanha a criança as etapas que devem ser seguidas. Por se tratar de uma técnica estéril, o procedimento se torna um pouco mais complexo do que pode parecer, porém o responsável pelo RN só irá realizar quando se sentir seguro e quando a equipe sentir que será capaz de garantir a segurança da criança

\section{Considerações finais}

A partir da análise do estudo, é notória a importância dos cuidados da enfermagem, pois é a equipe que a compõe que irá prestar a grande maioria da assistência ao paciente. O ambiente hospital gera angústia, medo e insegurança. Por isso, as consequências de um serviço mal elaborado serão devastadoras para o bem estar do paciente, que já se encontra em situação de vulnerabilidade.

Toda a equipe de saúde em si, de forma multidisciplinar, deve prestar o cuidado ao portador de mielomeningocele de maneira integral. A equipe médica é quem realiza o tratamento, pratica os cuidados cirúrgicos e propõe as intervenções que devem ser feitas diante do caso analisado. Já a equipe de fisioterapia atua na construção e na reabilitação das funções básicas do paciente, geralmente, as condutas serão tomadas após a alta hospitalar com respaldo médico. A equipe de enfermagem, além de dedicar ao paciente os cuidados físicos, é quem estabelece, em tempo integral, uma conexão com a criança e a família.

Sendo assim, fica claro, que a criança portadora de Mielomeningocele necessita de cuidados além dos básicos e, para isso, é imprescindível a presença dos profissionais de enfermagem para estabelecer a assistência necessária e também, construir uma relação de cuidado com o portador da MMC, com a família e com o profissional de saúde.

\section{Referências}

1. Freitas GL, de Sena RR, Silva JCF, Castro FFS. Reabilitação de crianças e adolescentes com mielomeningocele: o cotidiano de mães cuidadoras. Revista Gaúcha de Enfermagem, 2016 Dez.; 37(4):e60310.

2. Gurgel EPP, Rolim KMC, Galvão MTG, Cateano JA. Abordagem assistencial ao neonato portador de mielomeningocele segundo o modelo de adaptação de Roy. Revista da Escola de Enfermagem da USP, 2010, set.;44(3): 702-707.

3. Aguiar MJB, Campos AS, Aguiar RALP, Lana AMA, Magalhães RL, Babeto LT. Defeitos de fechamento do tubo neural e fatores associados em recém-nascidos vivos e natimortos. Jornal de Pediatria. (Rio J.). 2003, apr.; 79(2): 129-134.

4. Peralta, CFA. Prevenção dos defeitos de fechamento do tubo neural. Revista da Associação Médica Brasileira, 2001, dez.; 47(4): 276-277.

5. Gaiva MAM, Neves AQ, Siqueira FMG. O cuidado da criança com espinha bífida pela família no domicílio. Escola Anna Nery, 2009, dez.; 13(4): 717-725.

6. Silva PMV, dos Santos LP, Pereira Junior RS. Segurança do paciente e a assistência de enfermagem no cuidado intensivo. Revista Pró-UniverSUS, 2018 jan/jun.; 09(1): 81-85. 
7. Minayo MC de L (Org.) Pesquisa social: teoria, método e criatividade 19. Petrópolis: Vozes, 2014.

8. Programa Nacional Telessaúde (Rio Grande do Sul). Quais as causas e sintomas da hidrocefalia? 2010a. Disponível em: https://aps.bvs.br/aps/ quais-as-causas-e-sintomas-da-hidrocefalia/

9. Programa Nacional Telessaúde (Rio Grande do Sul). Quais as sequelas e complicações da hidrocefalia.2010b.Disponível em: https://aps.bvs.br/aps/ quais-as-sequelas-e-complicacoes-da-hidrocefalia/

10. Pereira ELR, Bichara CNC, Oliveira ANS, Costa MAT. Epidemiologia de pacientes com malformação de Chiari II internados no Hospital Fundação Santa Casa de Misericórdia do Pará. Rev. Para. Med., 2007 Jun; 21(2).

11. Salomão J, Francisco, BAR, Leibinger RD, Barbosa APA, Brandão MAPB. Malformação de Chiari do tipo II sintomática. Arq. Neuro-Psiquiatr. 1998 mar; 56(1): 98-106.

12. Azevedo MAJ, da Silveira SMML, Soler LMA. Promovendo o autocuidado: treinamento e assistência de enfermagem a pacientes portadores de bexiga neurogênica. Rev. Bras. Enferm. 1990 Dez; 43(1-2-3-4): 52-57.

13. Borelli, M. Pediatria Básica. Sarvier, São Paulo, 1968.

14. Rocha, FET, Gomes, CM. Bexiga neurogênica. Urologia fundamental. São Paulo: Planmark, 2010.

15. Brandão, AD, Fujisawa, DS, Cardoso, JR. Características de crianças com mielomeningocele: implicações para a fisioterapia. Fisioterapia em movimento. 2017 Jan; 22(1).

16. Façanha DMA. Avaliação da funcionalidade em crianças com mielomeningocele [Dissertação de Mestrado]. Fortaleza: Universidade Federal do Ceará; 2015.

17. Ministério da Saúde (BR). Atenção à Saúde do Recém-Nascido: guia para os profissionais de saúde. Brasília (DF); 2012.

18. Brasil. Ministério da Saúde (BR). Portaria n ${ }^{\circ} 930$ de 10 de maio de 2012. Define as diretrizes e objetivos para a organização da atenção integral e humanizada ao recém-nascido grave ou potencialmente grave e os critérios de classificação e habilitação de leitos de Unidade Neonatal no âmbito do Sistema Unico de Saúde (SUS). Diário Oficial da União. 11 mai 2012; Seção 1:138. Disponível em: http://bvsms.saude.gov.br/bvs/saudelegis/gm/2012/ prt0930_10_05_2012.html. 


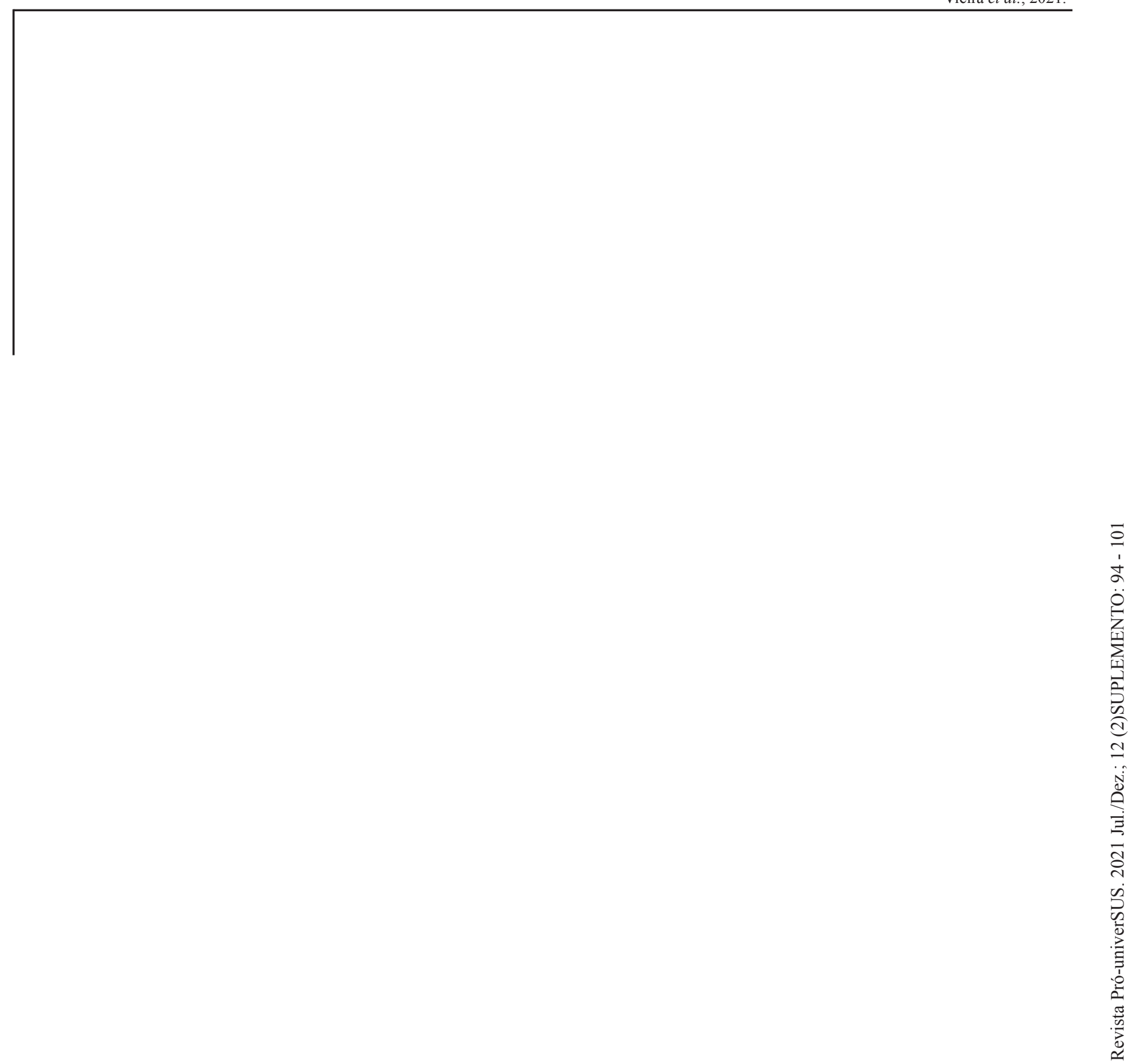




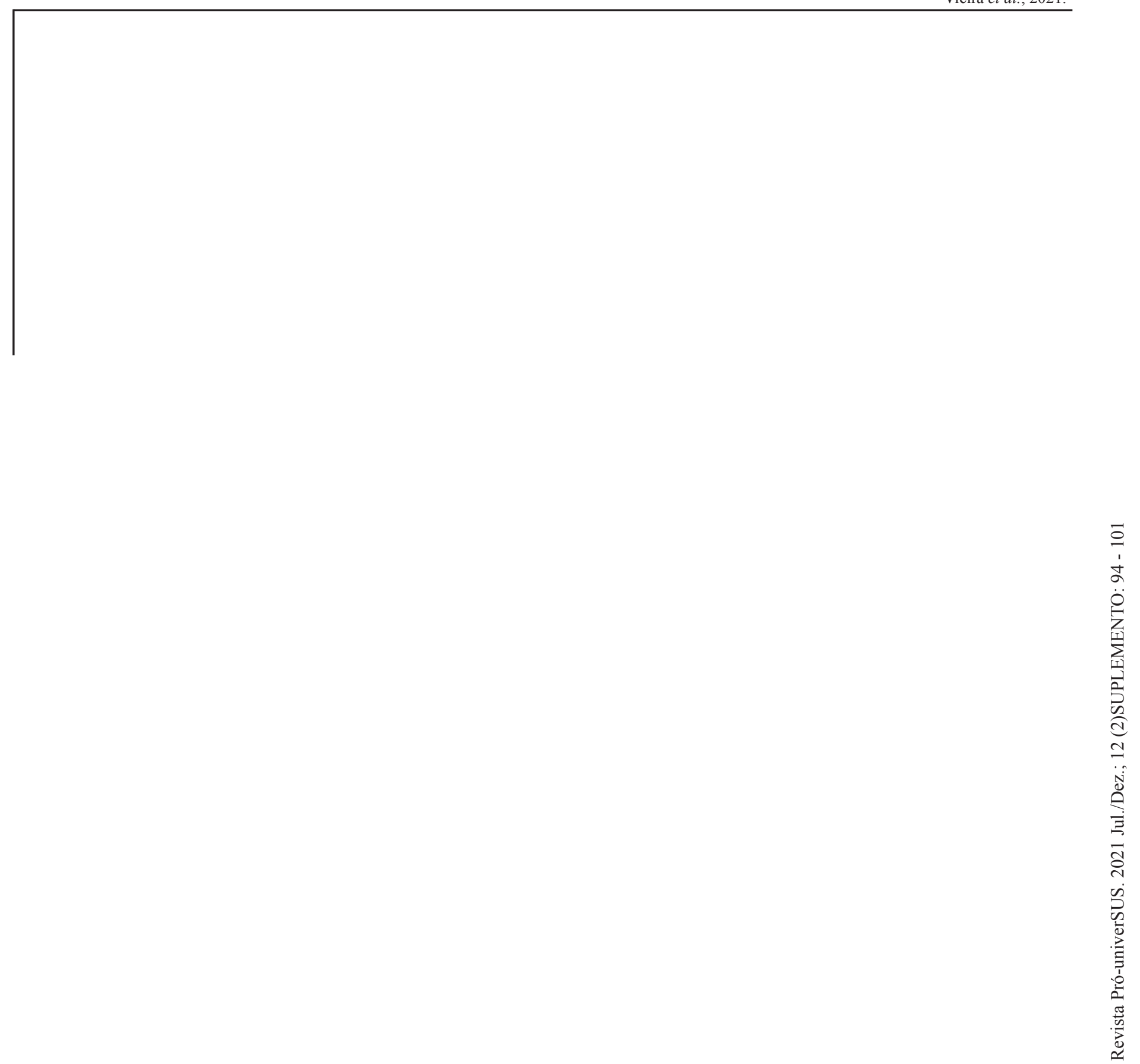

